\title{
Tegaderm CHG IV Securement Dressing for Central Venous and Arterial Catheter Insertion Sites: A NICE Medical Technology Guidance
}

\author{
Michelle Jenks $^{1} \cdot$ Joyce Craig $^{1} \cdot$ William Green ${ }^{1} \cdot$ Neil Hewitt $^{2} \cdot$ Mick Arber $^{1} \cdot$ \\ Andrew Sims ${ }^{3}$
}

Published online: 12 October 2015

(c) The Author(s) 2015. This article is published with open access at Springerlink.com

\begin{abstract}
Catheters are widely used for vascular access and for the administration of drugs or fluids in critically ill patients. This exposes patients to an infection risk. Tegaderm chlorhexidine gluconate (CHG) (developed by $3 \mathrm{M})$ a transparent securement dressing-covers and protects catheter sites and secures devices to the skin. It comprises a transparent adhesive dressing to act as a barrier against external contamination and an integrated gel pad containing an antiseptic agent. The Medical Technologies Advisory Committee (MTAC) at the National Institute for Health and Care Excellence (NICE) selected Tegaderm CHG for evaluation. One study was identified by the sponsor as relevant to the decision problem. From this, the sponsor concluded that compared with standard dressings, Tegaderm CHG is associated with lower rates of catheterrelated infection, but increased dermatitis incidence. The External Assessment Centre (EAC) identified four paired comparative studies between Tegaderm CHG, other CHG dressings or standard dressings. The EAC agreed with the sponsor's conclusion, finding that $\mathrm{CHG}$ dressings reduce infections compared with standard dressings. The sponsor constructed a de novo costing model. Tegaderm CHG
\end{abstract}

Michelle Jenks

michelle.jenks@york.ac.uk

1 York Health Economics Consortium, Enterprise House, Innovation Way, University of York, Heslington, York YO10 5NQ, UK

2 National Institute for Health and Care Excellence, Level 1A, City Tower, Piccadilly Plaza, Manchester M1 4BT, UK

3 Newcastle upon Tyne Hospitals NHS Foundation Trust, Freeman Hospital, Freeman Road, Newcastle upon Tyne NE7 7DN, UK generated cost savings of $£ 77.26$ per patient compared with standard dressings and was cost saving in $98.5 \%$ of a sample of sets of inputs (2013 prices). The EAC critiqued and updated the model's inputs, yielding similar results to those the sponsor estimate. The MTAC reviewed the evidence and decided to support the case for adoption, issuing a positive draft recommendation. After a public consultation, NICE published this as Medical Technology Guidance 25 .

\section{Key Points for Decision Makers}

The case for adopting the $3 \mathrm{M}$ Tegaderm chlorhexidine gluconate (CHG) IV securement dressing for central venous and arterial catheter insertion sites is supported by the evidence. This technology allows observation and provides antiseptic coverage of the catheter insertion site. It reduces catheter-related bloodstream infections and local site infections compared with semipermeable transparent (standard) dressings. It can be used with existing care bundles.

The 3M Tegaderm CHG IV securement dressing should be considered for use in critically ill adults who need a central venous or arterial catheter in intensive care or high-dependency units.

The estimated cost saving from using Tegaderm CHG instead of a standard dressing is $£ 73$ per patient. If this became standard practice, it has the potential to save the National Health Service (NHS) in England between $£ 4.2$ million and $£ 10.8$ million each year. 


\section{Introduction}

The National Institute for Health and Care Excellence (NICE) produces evidence-based medical technologies guidance to evaluate and, where appropriate, encourage adoption of novel and innovative medical devices and diagnostics within the National Health Service (NHS) in England. Sponsors of potentially eligible technologies notify their products to NICE's Medical Technologies Evaluation Programme (MTEP). Technologies are selected for evaluation as NICE medical technologies guidance by the MTEP if they have the potential to offer clinical benefits to patients and the NHS, and/or to reduce costs, compared with standard care.

Guidance is developed by the Medical Technologies Advisory Committee (MTAC) after the clinical and economic evidence submitted by the sponsor is assessed independently by an External Assessment Centre (EAC) and following a public consultation period. Devices and diagnostic tools with different, or complex, value propositions can be routed for evaluation through other NICE programmes, including the Diagnostics Assessment Programme. Campbell and Campbell [2] described the MTEP's methodology in more detail.

In July 2015, NICE issued final guidance on the Tegaderm chlorhexidine gluconate (CHG) dressing for use in intensive care settings with patients requiring an arterial or central venous catheter (CVC). Tegaderm $\mathrm{CHG}-\mathrm{a}$ transparent securement dressing-is used to cover and protect catheter sites and secure devices to the skin. The dressing, developed by $3 \mathrm{M}$, comprises a transparent adhesive dressing and an integrated gel pad. The gel pad absorbs fluid and contains an antiseptic agent consisting of $2 \% \mathrm{CHG}$, and the transparent adhesive dressing acts as a barrier against external contamination, protecting the catheter insertion site [3]. The EAC critiquing the evidence was the Newcastle upon Tyne Hospitals NHS Foundation Trust and York Health Economics Consortium partnership. Clinical experts, identified using NICE's published processes, provided advice to the EAC and MTAC.

This article is one of a series of Medical Technology Guidance summaries being published in Applied Health Economics and Health policy. It summarises the sponsor's submission on Tegaderm CHG's clinical and cost effectiveness, provides an overview of the EAC's report and subsequent development of the NICE guidance. Full documentation of the process, supporting evidence and the final guidance is on the NICE website [1].

\section{Background to the Indications and Device}

Critically ill adult patients are usually treated in intensive care units (ICUs) or high-dependency units (HDUs), to support the functioning of at least one organ. In England in $2012 / 2013$, there were 237,710 adult ICU episodes with organ support provided for a mean of 4 days [4]. Clinicians managing critically ill adult patients usually require vascular access for haemodynamic monitoring and/or the administration of medication or fluids, which is achieved through an arterial catheter or CVC. At least $78 \%$ of critically ill patients have some form of CVC [5].

The susceptibility of infection in critically ill patients is higher than the general population in part because ICU patients are exposed to specific risk factors, including invasive treatments and monitoring. Infections in critically ill patients can be further complicated because clinical signs may be masked by signs of co-existing disease [5].

Infections occur after catheters become colonised by microorganisms, which can take place during either catheter insertion or routine care. Colonisation can lead to catheter-related bloodstream infection (CRBSI) in an estimated 1.48 of every 1000 catheter days [6]. CRBSI occurs when bacteria or fungi present following colonisation, migrate along the extraluminal catheter surface and into the bloodstream [7]. This can lead to systemic infection, in turn causing an immune response that can lead to septic shock and multiple organ failure if undetected, increasing the risk of death [8].

Measures to reduce the risk of CRBSI and local infections at the catheter entry site are recommended in NICE guidelines on infection (Clinical Guideline 139). These include:

- Decontaminating the skin at the insertion site with CHG in $70 \%$ alcohol before inserting a central catheter.

- Using a sterile, transparent semipermeable membrane dressing to cover the insertion site.

- Changing the transparent semipermeable membrane dressing every 7 days, or sooner if the dressing is no longer intact or moisture collects under it.

- During dressing changes, the CVC insertion site and surrounding skin should be decontaminated, using CHG in $70 \%$ alcohol, and allowed to air dry [9].

Tegaderm $\mathrm{CHG}$ is an alternative to transparent semipermeable membrane dressings (referred to as 'standard dressings' herein). Antiseptic coverage is delivered through a CHG-impregnated gel incorporated into the dressing, which is placed over the catheter insertion site, and is intended to reduce skin and catheter colonisation, and hence the incidence of CRBSI. The gel is transparent, 
meaning the insertion site can be observed continually [3]. Tegaderm CHG was claimed to prevent catheter-related infection (CRI), thus reducing the risk of mortality, length of stay in ICU and costs associated with infection.

Tegaderm CHG was considered by the MTAC as an alternative to standard dressings used alone or in conjunction with a CHG sponge, as defined in the scope produced by NICE [10]. This describes the decision problem to be addressed by the sponsor and EAC and is described here in more detail.

\section{Decision Problem (Scope)}

\subsection{Population}

The population described in the scope was critically ill adult patients in ICUs or HDUs who require a CVC or arterial catheter. Although Tegaderm CHG is suitable for use in infants aged 2 months or above [3], children were outside of the scope due to a lack of evidence relating to the efficacy and safety of Tegaderm CHG in this subgroup.

\subsection{Intervention (Tegaderm CHG)}

The intervention specified in the scope was swabbing with $2 \%$ CHG in alcohol and Tegaderm CHG intravenous (IV) securement dressing. Tegaderm CHG is available in four sizes, with the most commonly used being $8.5 \times 11.5 \mathrm{~cm}$, containing $45 \mathrm{mg}$ of $\mathrm{CHG}$ [3]. All four sizes of the dressing are CE marked.

\subsection{Comparator (Current Practice)}

Two comparators were specified in the scope:

- Swabbing with $2 \% \mathrm{CHG}$ in alcohol, followed by sterile semipermeable transparent dressing (standard dressing).

- Swabbing with $2 \% \mathrm{CHG}$ in alcohol, followed by CHG-impregnated dressing (CHG sponge).

The CHG sponge comprises a standard dressing, together with a CHG-impregnated patch (marketed under the product name 'Biopatch'). Unlike Tegaderm CHG, it is not a single item, is not transparent and contains $18 \%$ dry CHG that is released by humidity from the skin.

\subsection{Outcomes}

The sponsor addressed six of the eight specified clinical outcomes for Tegaderm CHG and standard dressings, being CRBSI, skin and catheter colonisation, local site infection, dermatitis, adverse events and length of stay. Skin colonisation was the only outcome considered for the CHG sponge. The sponsor did not identify evidence on quality of life or mortality resulting from CRI.

CRBSI can be defined as a combination of one or more of the following, with no other infectious focus explaining the positive blood cultures:

- Positive peripheral blood cultures sampled immediately before or within $48 \mathrm{~h}$ after catheter removal.

- A positive quantitative catheter-tip culture.

- A blood-culture differential time-to-positivity of $2 \mathrm{~h}$ or more [11, 12].

Adverse events resulting from the use of both Tegaderm CHG and comparator dressings, including skin reactions or dermatitis, are described within clinical studies.

\section{Review of Clinical and Economic Evidence}

Clinical and economic evidence, including a de novo cost model, was submitted by the sponsor in line with the process set out by NICE [13]. The EAC critically appraised the submission and costing model against the scope. Section 4.1 summarises the clinical evidence submitted, the EAC's critique and the EAC's new work. Section 4.2 provides the same detail for the economic evidence.

\subsection{Clinical Effectiveness Evidence}

\subsubsection{Sponsor's Review of Clinical Effectiveness Evidence}

The sponsor searched for published clinical evidence relating to the decision problem. Included studies compared Tegaderm CHG with standard dressings or CHG sponges. The sponsor included one study [11] which reported a multicentre randomised control trial (RCT) of 1879 patients using 4163 catheters conducted between May 2010 and July 2011. Adult patients requiring intravascular access in 12 ICUs in hospitals in France were randomised to one of three groups: Tegaderm $\mathrm{CHG}$, standard dressing (Tegaderm Transparent Film Dressing) or highly adhesive dressing (Tegaderm HP Transparent Film Dressing). Assessors of suspected infection were blinded to dressing type. Patients had their skin prepared with alcohol-povidone iodine or alcohol-CHG $(0.5 \%)$. Dressings were replaced after $24 \mathrm{~h}$ and then every 3-7 days according to local protocol, or as required due to leaking or soiling. Patients with known allergies to $\mathrm{CHG}$ or transparent dressings were excluded. Results were reported up to $48 \mathrm{~h}$ after ICU discharge [11]. The sponsor appropriately critically appraised its included study [11], finding it to be at low risk of bias. 
An accurate description of the results reported in the RCT was provided by the sponsor. This included statistical comparisons between Tegaderm CHG and a combined control of standard dressings and highly adhesive dressings in line with those used in the study. Tegaderm CHG was reported to significantly reduce CRBSI compared with nonCHG dressings hazard ratio (HR) $=0.402$ [95\% confidence interval (CI) $0.186-0.868$ ]; $p=0.02\}$ and significantly reduce catheter colonisation $[\mathrm{HR}=0.412(95 \% \mathrm{CI}$ $0.306-0.556) ; p<0.0001]$. The length of stay in ICU was similar between groups (9 or 10 days). Severe contact dermatitis requiring removal of dressings occurred significantly more in the Tegaderm CHG group, compared with the non-CHG group (Tegaderm CHG $=1.1 \%$ of catheters; standard dressing $=0.1 \%$; highly adhesive dressing $=0.5 \% ; p<0.0001)$. No systemic adverse reactions were reported.

The sponsor provided US Food and Drug Administration (FDA) Manufacturer and User Facility Device Experience (MAUDE) and UK Medicines and Healthcare Products Regulatory Agency (MHRA) reports for Tegaderm CHG. The sponsor identified 109 FDA MAUDE reports, often describing local reactions occurring within $48 \mathrm{~h}$ of dressing application. Reactions included redness and irritation that was sometimes severe. In many cases, adverse reactions were self-healing. However, there were seven reports of an eschar (dead tissue) at the dressing site.

The sponsor reported, based on a study in healthy volunteers, that reduction in skin colonisation is similar between Tegaderm CHG and CHG sponges [14]. Further, the sponsor provided information on the relative ease of use and performance of the three dressings based on three studies that were identified during its clinical evidence review, but did not meet the inclusion criteria [15-17]. The patients in these studies were not all critically ill. Nurses within all studies reported statistically significantly higher satisfaction with Tegaderm CHG than standard dressings [15-17].

The sponsor concluded that the evidence shows, compared with standard dressings, Tegaderm CHG is associated with lower rates of CRBSI and catheter colonisation, but increased incidence of dermatitis. The sponsor stated that the results of its included study are likely to be generalisable to other settings with similar catheter care and dressing protocols.

\subsubsection{Critique of Clinical Effectiveness Evidence}

The EAC critiqued the sponsor's literature search, evidence selection and quality assessment of included studies. Replication of the sponsor's searches obtained a similar yield of search results. The study selection criteria employed by the sponsor included only comparative studies containing a Tegaderm CHG arm. Although consistent with the decision problem issued by NICE, this meant that no CHG sponge evidence was included. The EAC reviewed the critical appraisal and results reported by the sponsor [11], finding these to be detailed and accurate.

The EAC undertook its own literature search and included studies comparing any two of the three dressing types in order to identify relevant studies of CHG sponges to inform the comparator. A search comprising three concepts (catheters, dressings and $\mathrm{CHG}$ ) was carried out on a number of databases, including MEDLINE, Embase and the Cochrane Library, and the grey literature (strategies are described in the EAC assessment report [1]).

Included studies were limited to prospective comparative studies of critically ill adult patients in ICUs or HDUs. The selection criteria were in line with the scope issued by NICE, with the exception of the skin preparation solution. The scope specifies that prior to catheter insertion, skin should be prepared with $2 \%$ CHG in alcohol solution as defined in the NICE clinical guideline on infection [9]. In order to maximise sensitivity, the skin preparation solution was not specified within the selection criteria.

The PRISMA diagram [18] (Fig. 1) shows the number of papers retrieved and excluded following dual selection by two independent reviewers, together with reason for exclusion. The EAC included four studies; two compared Tegaderm CHG with standard dressings $[11,19]$ and two compared CHG sponges with standard dressings $[12,20]$. One of the two Tegaderm CHG studies was presented as a poster after the sponsor's clinical evidence submission [19]. The studies comprised the following number of patients: $N=1879$ [11]; $N=1653$ [12]; $N=273$ [19] and $N=33$ [20]. Three studies were published RCTs [11, $12,20]$, and the remaining one study was a comparative observational study [19]. Two of the three RCTs recruited sufficient patient numbers to achieve $80 \%$ statistical power $[11,12]$, whilst the third study was underpowered because of limited resources precluding the ability to recruit the number of patients required to achieve statistical significance [20]. The study reporting skin colonisation in healthy volunteers was excluded by the EAC given no critically ill patients were included [14].

Data from the included studies were extracted by one reviewer and checked by a second. There were no significant differences in the baseline characteristics of patients in each group within each study. All four studies recruited critically ill adult patients requiring intravascular access within an ICU or critical care unit setting. CVCs were included in all four studies, and arterial catheters were also included in two of the three RCTs [11, 12]. The same two studies excluded patients with a known allergy to CHG or transparent dressings and reported that microbiologists processing the skin and catheter cultures were blinded to 
Fig. 1 PRISMA flow diagram showing clinical studies assessed during the External Assessment Centre review

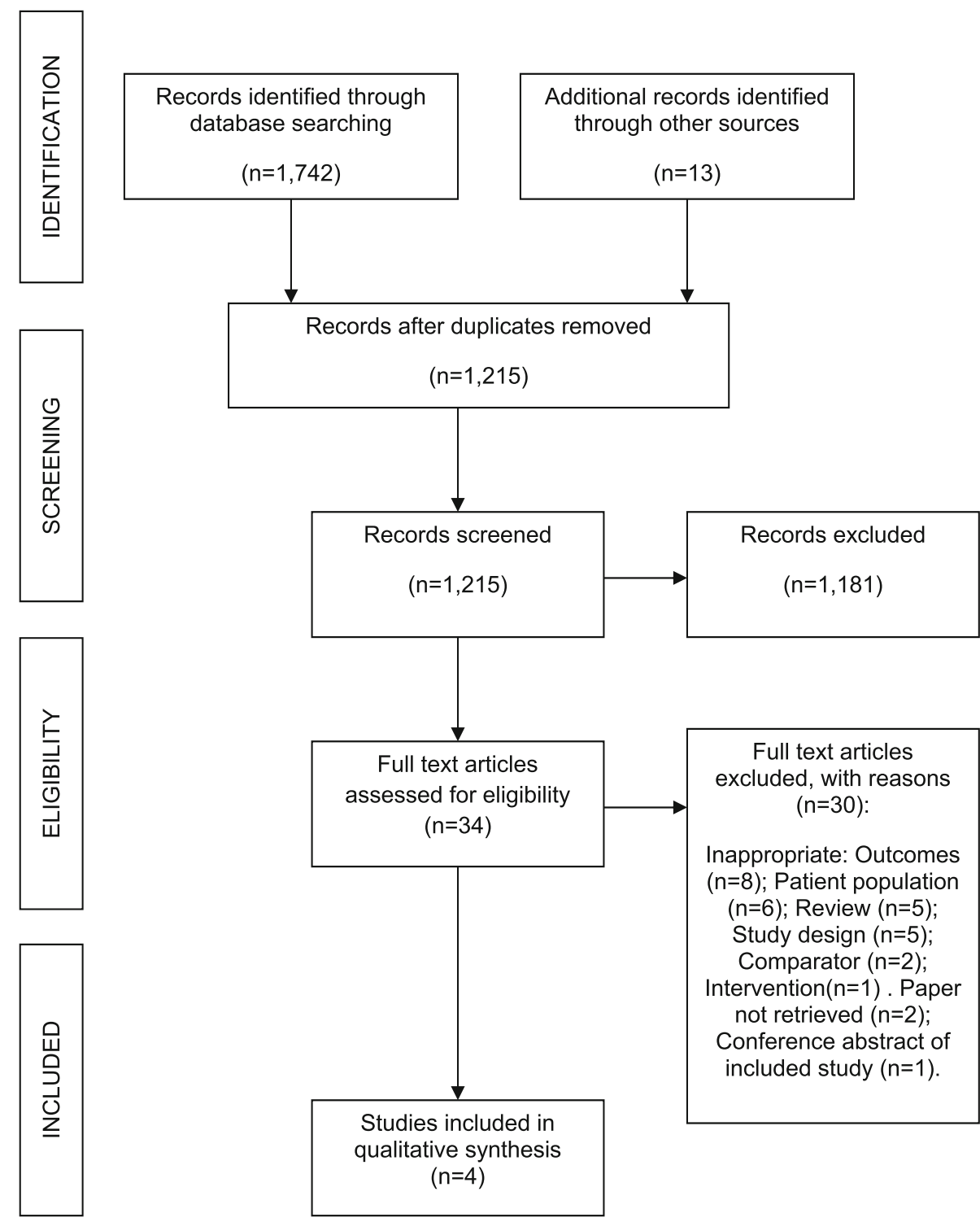

provided in the MTEP sponsor submission template was undertaken for three studies $[11,12,20]$, but was not possible for the study published as a conference poster [15], because of limited information. Two of the three RCTs were well conducted and judged to be at low risk of bias, with the only concern being that no reason was provided for the exclusion of around 150 patients from the two studies ( $n=156$ [11] and $n=147$ [12]). If these patients were inherently different to those included, there is risk of bias. The third RCT [20] provided insufficient information relating to the methodology to rule out bias.

The sponsor's results included tests for differences in outcomes between Tegaderm CHG and a combined control of standard dressings and highly adhesive dressings. The sponsor advised that Tegaderm highly adhesive dressings (Tegaderm HP Transparent Film Dressing) are not used within England, but are standard care within France where 
Table 1 Summary of critical appraisal in relation to decision problem

\begin{tabular}{|c|c|c|c|}
\hline Study & Internal validity & External validity & $\begin{array}{l}\text { Usefulness to decision } \\
\text { problem }\end{array}$ \\
\hline $\begin{array}{l}\text { Timsit et al., } \\
\text { Tegaderm CHG vs. } \\
\text { standard dressing } \\
\text { [11] }\end{array}$ & $\begin{array}{l}\text { Weaknesses in a number of domains were unlikely } \\
\text { to introduce bias }\end{array}$ & $\begin{array}{l}\text { Treatment regime and patient } \\
\text { characteristics are partially } \\
\text { applicable to scope }\end{array}$ & $\begin{array}{l}\text { High, most relevant and } \\
\text { highest quality study } \\
\text { on Tegaderm CHG }\end{array}$ \\
\hline $\begin{array}{l}\text { Timsit et al., CHG } \\
\text { sponge vs. standard } \\
\text { dressing [12] }\end{array}$ & $\begin{array}{l}\text { Weaknesses in a number of domains were unlikely } \\
\text { to introduce bias }\end{array}$ & $\begin{array}{l}\text { Treatment regime and patient } \\
\text { characteristics are partially } \\
\text { applicable to scope }\end{array}$ & $\begin{array}{l}\text { High, most relevant and } \\
\text { highest quality study } \\
\text { on CHG sponge }\end{array}$ \\
\hline $\begin{array}{l}\text { Roberts and Cheung, } \\
\text { CHG sponge vs. } \\
\text { standard dressing } \\
{[20]}\end{array}$ & $\begin{array}{l}\text { Generally poor across all domains and poorly } \\
\text { reported. Potential for: } \\
\text { Selection bias as randomisation method not } \\
\text { described } \\
\text { Performance bias from limited information on } \\
\text { participants } \\
\text { Detection bias as no blinding was reported }\end{array}$ & $\begin{array}{l}\text { Treatment regime is partially } \\
\text { applicable to scope. Insufficient } \\
\text { information on patient } \\
\text { characteristics }\end{array}$ & Low \\
\hline $\begin{array}{l}\text { Karpanen et al., } \\
\text { Tegaderm CHG vs. } \\
\text { standard dressing } \\
{[19]}\end{array}$ & $\begin{array}{l}\text { Certain weaknesses noted, e.g. observational so no } \\
\text { randomisation. Overall, difficult to assess as } \\
\text { results are from a conference poster only }\end{array}$ & $\begin{array}{l}\text { NHS treatment regime is applicable } \\
\text { to scope. Lack of information on } \\
\text { patient characteristics }\end{array}$ & Medium \\
\hline
\end{tabular}

$C H G$ chlorhexidine gluconate, $N H S$ National Health Service

the study was set. The results comparing Tegaderm CHG with standard dressings have greater applicability to the decision problem than those including highly adhesive dressings. However, the EAC noted many of the comparisons were only between dressings containing $\mathrm{CHG}$ versus dressings not containing CHG.

The results of the EAC's four included studies [11, 12, 19, 20] are provided in Table 2. Both studies by Timsit et al. reported a statistically significant decrease (at $p<0.05)$ in CRBSI with CHG-impregnated dressings (Tegaderm CHG or CHG sponge) [11, 12].

Catheter colonisation rates were reported in all studies $[11,12,19,20]$, and skin colonisation rates were reported in one study [19]. Both studies comparing Tegaderm CHG to standard dressings reported lower catheter colonisation rates. Timsit et al. [11] reported a lower incidence of catheter colonisations with Tegaderm CHG than standard dressings of 9.6 versus 4.3 per 1000 catheter days ( $p<0.0001$ for the combined control group). Karpanen et al. [19] reported a lower CVC intradermal section colonisation incidence $(p=0.037)$ and a lower incidence of positive CVC tip colonisation $(p<0.05)$ with Tegaderm CHG. Timsit et al. [12] found catheter colonisation rates to be lower in the CHG sponge group compared with standard dressing (6.3 per 1000 catheter days vs. 15.8 per 1000 catheter days, $p<0.001)$. Roberts and Cheung [20] reported a non-significant higher incidence of both catheter and skin colonisation with $\mathrm{CHG}$ sponge compared with standard dressings.

No systemic adverse reactions to $\mathrm{CHG}$ were reported in any study $[11,12,19,20]$; two studies explicitly stated no systemic adverse reactions had occurred [11, 12]. Both studies excluded patients with known allergies to $\mathrm{CHG}$, which may limit the generalisability of adverse reaction results to the NHS. Severe contact dermatitis requiring removal of the dressing was reported in two studies [11, 12]. In both studies, the incidence of dermatitis was higher with CHG-impregnated dressings $(p<0.0001$ [11] and $p=$ not reported [12]).

The EAC analysed data from FDA MAUDE for Tegaderm CHG and its comparators, finding that following launch of an improved breathable version of Tegaderm CHG (in 2010), the rate of reported adverse reactions had fallen. Reports for both Tegaderm CHG and CHG sponge (specifically Biopatch) were similar in terms of the types and volume of reactions being described: largely mild, selfhealing skin reactions requiring removal of the dressing.

The EAC validated the information provided by the sponsor on the relative ease of use and performance of Tegaderm CHG through targeted searching and seeking expert opinion. Evidence from three US studies of nurses managing non-critically ill patients reported Tegaderm CHG is rated more favourable to use than $\mathrm{CHG}$ sponges $(p<0.05)$ in terms of ease of application and removal, ability to see IV site and ease of training [21-23]. There were mixed results in terms of nurse satisfaction with ease of correct application, transparency (site visibility), ease of dressing removal and patient discomfort. However, differences rarely reached significance [21-23]. Evidence from three studies comparing Tegaderm CHG to standard dressings found nurses to be significantly more satisfied with Tegaderm CHG (at $p<0.05$ ) as it provided more 


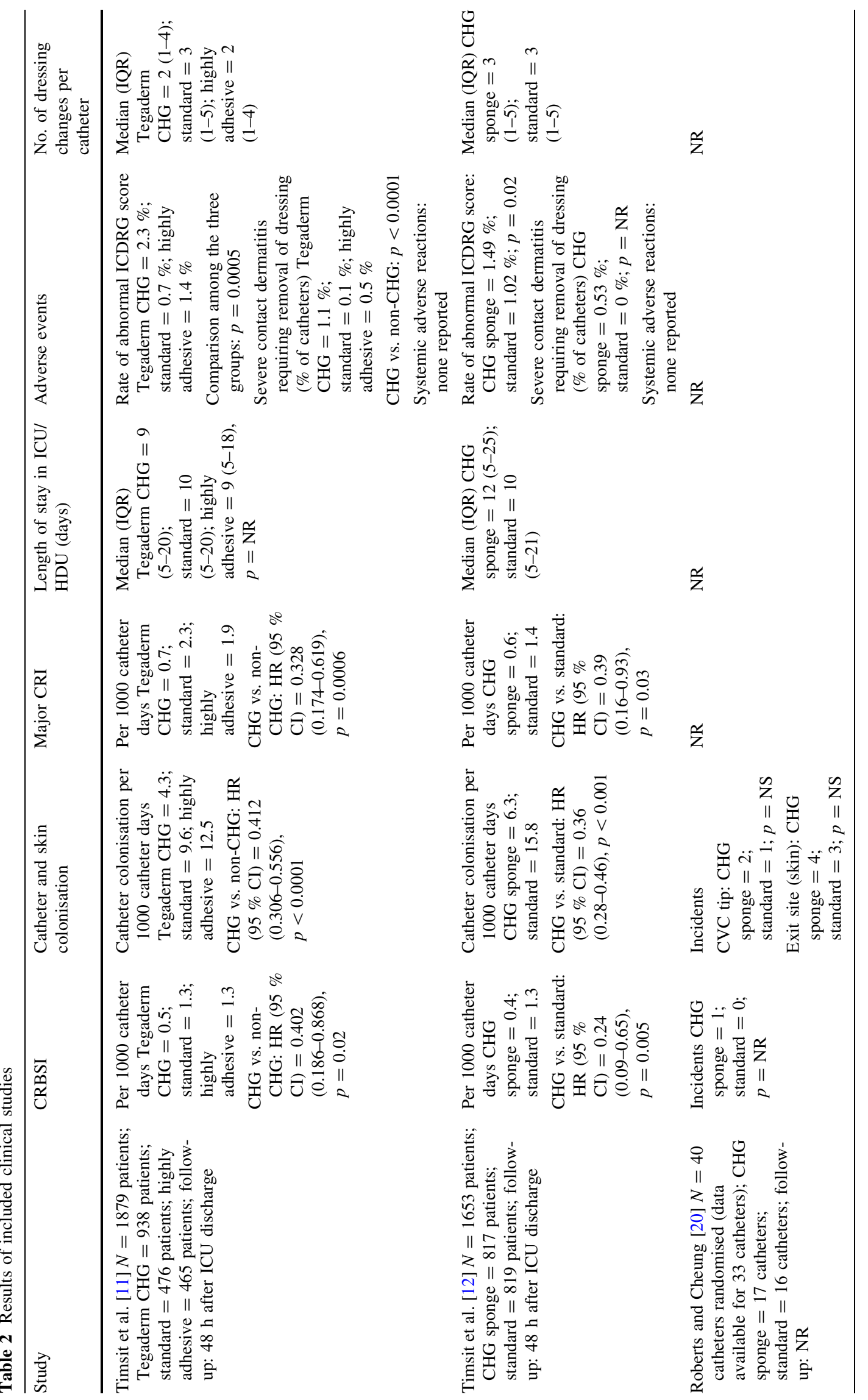




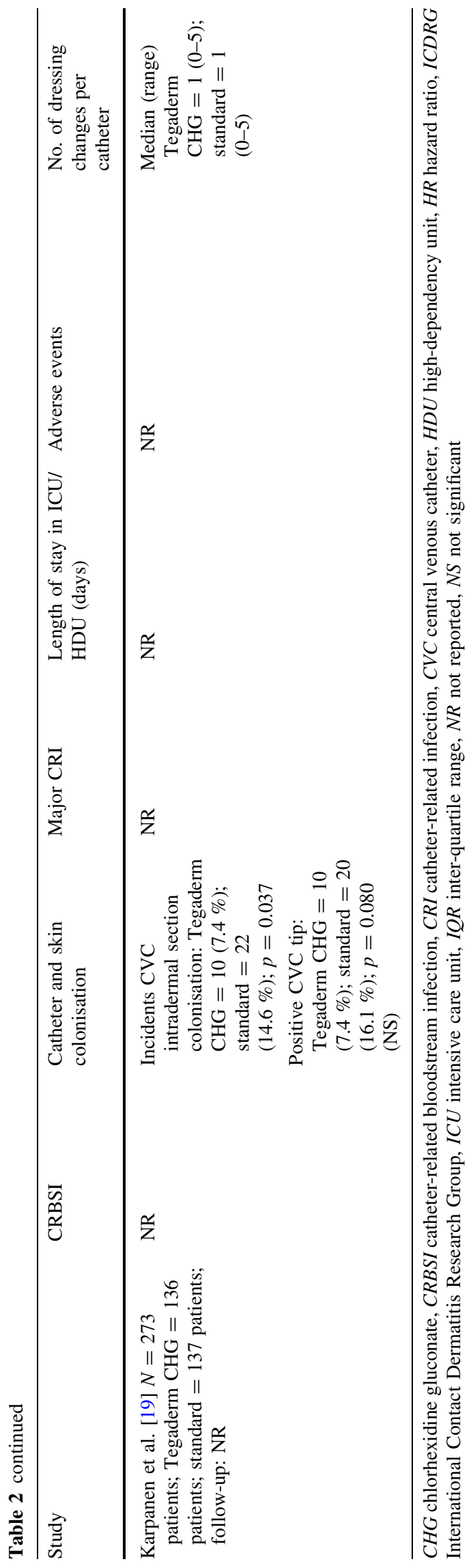

satisfactory securement and was easier to apply [15-17]. Expert opinion supported the view that Tegaderm CHG is easier to use than CHG sponges and is at least as easy to use as standard dressings.

No evidence was identified relating to mortality from CRI, local site infections or quality of life. Clinical experts advised that CRBSI can have a devastating impact on mortality and quality of life.

\subsubsection{New Work Undertaken by EAC Relating to Clinical Evidence}

The EAC performed a $Z$ test to estimate whether the CRBSI rate reported for Tegaderm CHG was significantly different to that reported for the CHG sponge. The total number of catheter days for each treatment group was obtained from the study authors in order to conduct the $Z$ test $[11,12]$. A score of 0.56 was obtained, with a $p$ value of 0.58 . Therefore, no statistically significant difference is estimated in the CRBSI rate between Tegaderm CHG and the CHG sponge, suggesting that where baseline CRBSI rates are at 1.3 per 1000 catheter days (as in the two RCTs by Timsit et al. [11, 12]), both Tegaderm CHG and CHGimpregnated sponges are effective in reducing CRBSI. There is insufficient evidence to demonstrate that one is more effective than the other.

\subsubsection{Conclusions from Clinical Effectiveness Evidence}

The EAC did not identify any evidence to suggest that the conclusions drawn by the sponsor are invalid. Furthermore, consideration of studies comparing CHG sponges to standard dressings suggested that the rates of CRBSI and surrogate measures of infection, such as catheter colonisation, are likely to be similar with CHG sponges and Tegaderm CHG. The EAC concluded that both types of CHG-impregnated dressings (Tegaderm CHG or CHG-impregnated sponge) lead to lower rates of CRBSI and catheter colonisation than standard dressings. There is a higher risk of dermatitis with both Tegaderm CHG and CHG sponges than with standard dressings, although this risk has declined with the modified Tegaderm CHG product. Users of the dressings reported that Tegaderm CHG is at least as easy to use as standard dressings and easier to use than the CHG sponge due to its transparency and all-in-one component.

\subsection{Economic Evidence}

\subsubsection{Sponsor's Economic Submission}

The sponsor undertook a search for economic studies of interventions aiming to reduce CRI. Five studies were 
included by the sponsor that reported on the cost-benefits of CHG sponges or antiseptic catheters compared with standard care [24-28]. The sponsor drew no conclusions from the economic evidence, other than that no UK-based cost-effectiveness studies comparing Tegaderm CHG to standard dressing were available.

A de novo decision analytic model was submitted by the sponsor, which compared Tegaderm $\mathrm{CHG}$ and standard dressings over a short time horizon, from an NHS and Personal Social Services perspective, with prices expressed at 2013 levels. Given the lack of clinical evidence comparing Tegaderm CHG with the CHG sponge, the sponsor excluded this comparator from its analysis. A fully executable model was created in Microsoft Excel ${ }^{\circledR}$. In the model, a simulated cohort of critically ill patients requiring intravascular access received a Tegaderm CHG dressing or a standard dressing. Patients within the model were at risk of CRBSI, local site infection or dermatitis (Fig. 2). A baseline risk of each complication was used for standard dressings and relative risks applied to these for Tegaderm CHG. The sponsor's base case results were probabilistic, based on 1000 iterations of the model.

The model was populated using data identified from the sponsor's clinical evidence review of the effectiveness of Tegaderm CHG on CRBSI, local site infection and dermatitis [11]. The economic literature review identified studies with evidence of baseline risk of complications, cost and resource use inputs [25, 26, 28], baseline CRBSI risk [6] and the number of dressings required [3]. All model input parameters are shown in Table 3.

Probabilistic results from the sponsor's economic model showed Tegaderm CHG generates cost savings of $£ 77.26$ per patient compared with standard care. This was based on costs of $£ 99.63$ per patient using Tegaderm CHG and $£ 176.89$ per patient using standard dressings. Probabilistic sensitivity analysis (PSA) was conducted using the ranges and distributions shown in Table 3. Tegaderm CHG was found to be cost saving in $98.5 \%$ of model runs. Univariate sensitivity analyses were conducted around the two key drivers of the results: baseline risk of CRBSI (0.5-5.5 infections per 1000 catheter days) and the cost of CRBSI (£5000-15,000). Tegaderm CHG remained cost saving compared with standard dressings for all values examined.

The results of the de novo economic modelling led the sponsor to conclude that the potential cost savings generated by Tegaderm CHG compared with standard dressings are robust to the uncertainties identified, particularly the rate of CRBSI and its associated costs. These were also consistent with cost savings generated by other

Fig. 2 Economic model structure

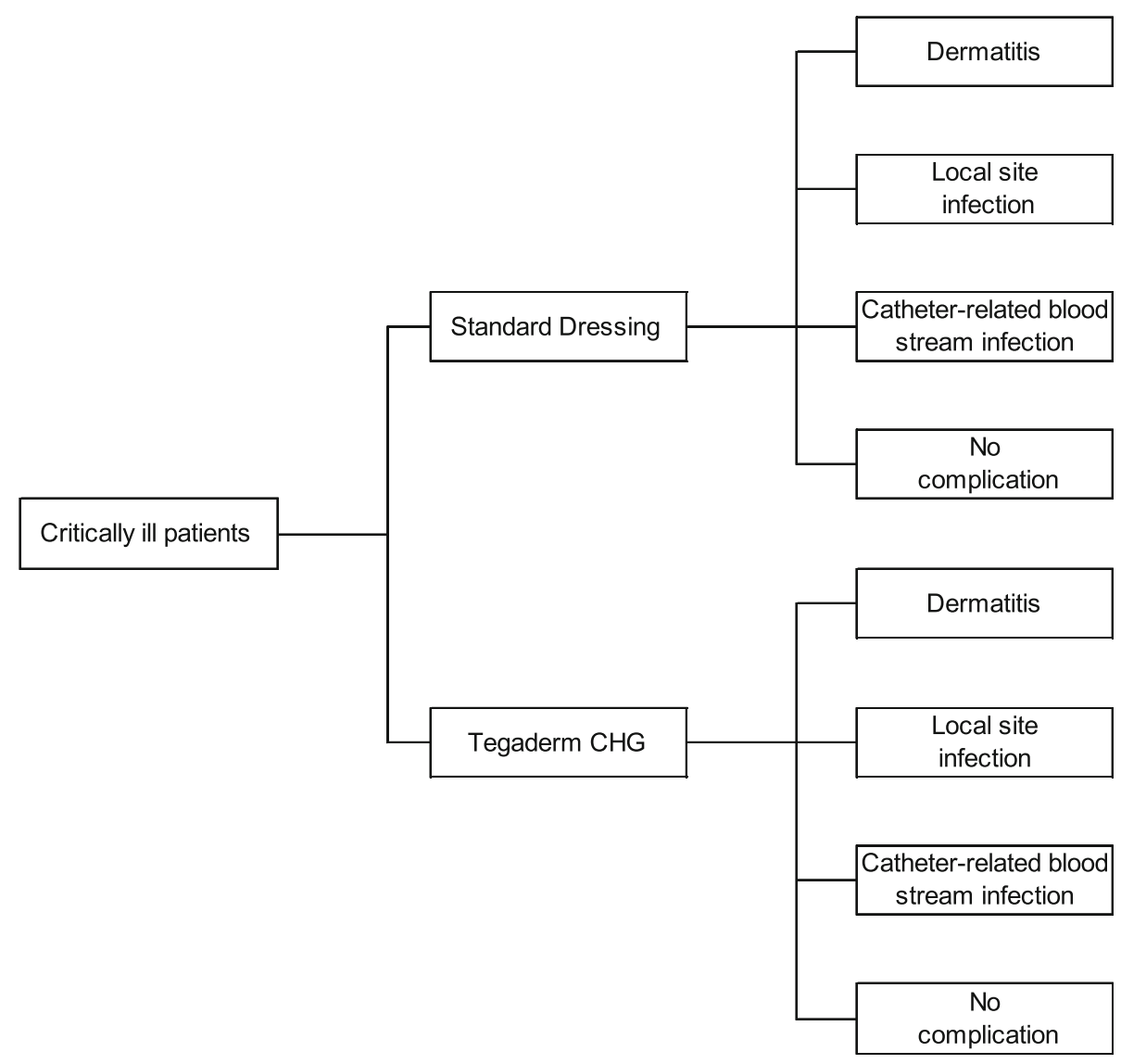


Table 3 Model input parameters used in sponsor's economic model

\begin{tabular}{|c|c|c|}
\hline Parameter & Value, range and distribution & Description \\
\hline \multicolumn{3}{|l|}{ Clinical input parameters } \\
\hline Baseline CRBSI rate & $\begin{array}{l}1.48 \text { per } 1000 \text { catheter days; normal distribution; } \\
\text { standard error }=0.074 \text { (calculated in Excel sheet } \\
\text { as mean divided by } 20 \text { ) }\end{array}$ & $\begin{array}{l}\text { Source was data reported in Matching Michigan study, based } \\
\text { on CRBSI rates reported in } 97 \% \text { of English NHS ICUs [6] }\end{array}$ \\
\hline $\begin{array}{l}\text { Hazard ratio for CRBSI } \\
\text { with Tegaderm CHG }\end{array}$ & $\begin{array}{l}\text { 0.402; lognormal distribution; Alpha }=-0.911 \\
\text { (calculated in Excel sheet as log mean); } \\
\text { Beta }=-0.393 \text { (source unclear) }\end{array}$ & $\begin{array}{l}\text { Source was the RCT identified in the sponsor's clinical } \\
\text { review [11], and hazard ratio was applied to CRBSI } \\
\text { baseline risk }\end{array}$ \\
\hline $\begin{array}{l}\text { Baseline local siteI } \\
\text { infection rate }\end{array}$ & $\begin{array}{l}0.1 \text { per patient; normal distribution; standard } \\
\text { error }=0.01 \text { (calculated in Excel sheet as mean } \\
\text { divided by } 10 \text { ) }\end{array}$ & $\begin{array}{l}\text { Source was a cost-benefit analysis identified in the sponsor's } \\
\text { cost-effectiveness review [28]. The original source was a } \\
\text { small US RCT published in } 1996 \text { [34] }\end{array}$ \\
\hline $\begin{array}{l}\text { Hazard ratio for localI } \\
\text { site infection withI } \\
\text { Tegaderm CHG }\end{array}$ & $\begin{array}{l}\text { 0.402; lognormal distribution; Alpha }=-0.911 \\
\text { (calculated in Excel sheet as log mean); } \\
\text { Beta }=-0.393 \text { (source unclear) }\end{array}$ & $\begin{array}{l}\text { Assumed to be the same as the hazard ratio for CRBSI [11] } \\
\text { and applied to baseline risk }\end{array}$ \\
\hline Baseline dermatitis risk & $\begin{array}{l}0.0026 \text { per catheter; normal distribution; standard } \\
\text { error }=0.00026 \text { (calculated in Excel sheet as mean } \\
\text { divided by 10) }\end{array}$ & $\begin{array}{l}\text { Source was a cost-benefit analysis identified in the sponsor's } \\
\text { cost-effectiveness review [26]. The original source was the } \\
\text { RCT by Timsit et al. comparing CHG sponge with standard } \\
\text { dressings [12] }\end{array}$ \\
\hline $\begin{array}{l}\text { Relative risk forI } \\
\text { dermatitis withI } \\
\text { Tegaderm CHG }\end{array}$ & $\begin{array}{l}\text { 4.4; lognormal distribution; Alpha }=18.034 \\
\text { (calculated in Excel sheet as log mean); } \\
\text { Beta }=-0.393 \text { (source unclear) }\end{array}$ & $\begin{array}{l}\text { Relative risk was taken from the RCT identified in the } \\
\text { sponsor's clinical review [11] and applied to the baseline } \\
\text { risk }\end{array}$ \\
\hline \multicolumn{3}{|c|}{ Cost and resource use input parameters } \\
\hline Cost of CRBSI & $\begin{array}{l}£ 9,990 ; \text { Gamma distribution; Alpha }=198 \\
\text { (calculated in Excel sheet as mean divided by } \\
\text { Beta); Beta }=50 \text { (assumption) }\end{array}$ & $\begin{array}{l}\text { Source was a } 2008 \text { health technology assessment identified in } \\
\text { the sponsor's cost-effectiveness review [25] and validated } \\
\text { using expert advice by the sponsor }\end{array}$ \\
\hline Cost of dermatitis & $\begin{array}{l}£ 150 \text {; Gamma distribution; Alpha }=30 \text { (calculated } \\
\text { in Excel sheet as mean divided by Beta); } \\
\text { Beta }=5 \text { (assumption) }\end{array}$ & $\begin{array}{l}\text { Source was a cost-benefit analysis identified from the } \\
\text { sponsor's cost-effectiveness review [26]. The cost includes } \\
\text { replacement of the catheter }\end{array}$ \\
\hline $\begin{array}{l}\text { Cost of local siteI } \\
\text { infection }\end{array}$ & $\begin{array}{l}£ 250 \text {; Gamma distribution; Alpha }=50 \text { (calculated } \\
\text { in Excel sheet as mean divided by Beta); } \\
\text { Beta }=5 \text { (assumption) }\end{array}$ & $\begin{array}{l}\text { Source was a study identified in the sponsor's cost- } \\
\text { effectiveness analysis [28]. The original source of the cost } \\
\text { provided no additional information [39] }\end{array}$ \\
\hline $\begin{array}{l}\text { Cost of TegadermI } \\
\text { CHG }\end{array}$ & $£ 6.21$; value is fixed & $\begin{array}{l}\text { Published price of the most commonly used size of dressing: } \\
8.5 \mathrm{~cm} \times 11.5 \mathrm{~cm} \text { (catalogue number 1657R) }\end{array}$ \\
\hline $\begin{array}{l}\text { Cost of standardI } \\
\text { dressing }\end{array}$ & $£ 1.34$; value is fixed & $\begin{array}{l}\text { The sponsor obtained this cost from their own prices for } \\
\text { Tegaderm IV catalogue number } 1635\end{array}$ \\
\hline $\begin{array}{l}\text { Number of days withI } \\
\text { catheter }\end{array}$ & $\begin{array}{l}10 \text { days; normal distribution; standard } \\
\text { error }=2 \text { days (calculated in Excel sheet as mean } \\
\text { divided by } 5 \text { ) }\end{array}$ & $\begin{array}{l}\text { Source was a study identified in the sponsor's cost- } \\
\text { effectiveness analysis [28]. The original source of the value } \\
\text { provided no additional information [40] }\end{array}$ \\
\hline Number of dressings & $\begin{array}{l}\text { 3; normal distribution; standard error }=0.3 \\
\text { (calculated in Excel sheet as mean divided by } 10 \text { ) }\end{array}$ & $\begin{array}{l}\text { Estimate was a conservative assumption based on to } \\
\text { Tegaderm CHG Instructions for Use and expert opinion }\end{array}$ \\
\hline
\end{tabular}

CHG chlorhexidine gluconate, CRBSI catheter-related bloodstream infection, ICU intensive care unit, NHS National Health Service, RCT randomised control trial

antimicrobial devices used to prevent CRI, from the literature [24-28].

\subsubsection{Critique of Economic Evidence}

The EAC was unable to replicate the sponsor's literature search for economic evidence as insufficient information was provided. The EAC excluded the five studies included by the sponsor because none of these used Tegaderm $\mathrm{CHG}$ as a comparator [24-28]. Rather, these studies all compared the cost effectiveness of CHG sponges with standard dressings.
The EAC's search for clinical evidence did not specify study design; therefore, the results were sifted for any economic studies relating to Tegaderm CHG. Four cost-benefit analyses, published subsequent to the conduct of the sponsor's literature search, were included [29-32]. All were conference abstracts, written by the same authors, using data from the Timsit et al. [11] RCT. Each study used different economic model structures and/or reported different results, in order to assess the cost-benefits of Tegaderm CHG compared with standard dressings from a French healthcare system perspective. None of the studies reported statistically significant differences in outcomes between the comparators. 
The EAC judged as accurate the sponsor's conclusion that no UK-based cost-effectiveness studies comparing Tegaderm CHG to standard dressing were available. Thus, it was appropriate for the sponsor to build a de novo economic model. The EAC replicated the sponsor's calculations in order to confirm their accuracy, correcting a minor error relating to the dermatitis rate in both arms of the model. A number of structural assumptions were identified by the EAC; these simplifying assumptions were necessary given the data available and were unlikely to have introduced material bias. The EAC judged, after seeking advice from clinical experts, that the structure of the model, whilst relatively simple, captured the key differences between Tegaderm CHG and standard dressings. Ideally, however, the model would also have included the third dressing type, the CHG sponge. The sponsor judged that the lack of comparative data between Tegaderm $\mathrm{CHG}$ and $\mathrm{CHG}$ sponge precluded the inclusion of this comparison in its cost-effectiveness analysis. The exclusion of this dressing from any cost analysis was the key weakness of the sponsor's economic submission.

All model inputs were validated by the EAC using expert advice and the literature. The EAC made changes to a number of model inputs, as shown in Table 4. Both the baseline rate and relative risk of dermatitis were amended, such that the baseline rate used was from the standard dressing arm of an RCT [11] and the relative risk was assumed to be 1 given the low rate of dermatitis with the new design of Tegaderm CHG. The baseline local site infection rate was updated to the 2013 rate from NHS ICUs in Wales [33], rather than the 1996 value used by the sponsor [34]. The cost of dermatitis and local site infections were lowered based on expert advice and the costs of both dressings updated to NHS Supply Chain prices [35]. In addition, the EAC amended distributions and ranges around input parameters to those it deemed most appropriate (see Table 4).

Following these updates, the EAC re-ran the sponsors model 10,000 times (based on the number of iterations required for results to become stable). Tegaderm CHG generated cost savings of $£ 72.90$ per patient compared with standard dressings and was cost saving in $97.8 \%$ of runs. Univariate sensitivity analyses using the ranges specified for each model input parameters did not change the direction of the results.

\subsubsection{New Work Undertaken by EAC Relating to Economic Evidence}

The EAC considered a scenario using an alternative baseline CRBSI rate. The rate used in the sponsor's and EAC's base case analyses of 1.48 CRBSI per 1000 catheter days was taken from NHS ICUs in 2010, reported in the
Matching Michigan study [6]. Before and during this study, infection rates trended downwards; thus the current CRBSI rate may differ to that from 2010. In 2013, NHS Scotland and NHS Wales reported annual confirmed CRBSI rates of 0.3 per 1000 catheter days (95\% CI $0.2-0.6$ ) [36] and 0.19 per 1000 catheter days [33], respectively, although NHS Scotland acknowledged that CRBSIs were potentially underreported. This rate was considered in a scenario to explore the impact on cost effectiveness in ICUs with low CRBSI rates. In this scenario, Tegaderm CHG was cost saving compared with standard dressings in $57.9 \%$ of the 10,000 model iterations, generating an average saving of $£ 3.56$ per patient. Univariate sensitivity analysis showed that Tegaderm CHG became cost incurring where the HR of CRBSI to standard dressing was 0.53 or above, or the cost of each CRBSI was $£ 8,000$ or below.

The EAC considered the relative cost effects of Tegaderm $\mathrm{CHG}$ and $\mathrm{CHG}$ sponges, agreeing with the sponsor that the lack of comparative data between Tegaderm CHG and CHG sponge makes an analysis difficult. However, some exploratory analysis was conducted. The EAC concluded that based on the available clinical evidence and the $\mathrm{Z}$ score generated, there appeared to be no significant differences in the clinical efficacy of the two CHG-impregnated dressings. Clinical experts advised that the resource implications of the two dressings are similar, although potentially higher with the CHG sponge because of its two-component nature. Therefore, it was assumed that clinical efficacy and resource use between the two dressings are essentially equivalent, enabling a cost-minimisation exercise to be conducted.

The NHS Supply Chain catalogue price for a CHGimpregnated dressing (Biopatch) is $£ 6.80$ [35]. Adding a standard dressing gives a combined cost of $£ 8.13$, around $£ 2$ per dressing more than Tegaderm CHG. The sponsor provided a lower cost for Biopatch of $£ 5.16$. Given that no sales of Biopatch were made through NHS Supply Chain in the two previous financial years, but clinicians advised they use the dressing, it is likely that Trusts purchase the dressing via other sources at a cheaper price than the NHS Supply Chain listed price [35]. Using the $£ 5.16$ cost for Biopatch results in a combined cost, with the standard dressing, of $£ 6.49$ per dressing, slightly more expensive than Tegaderm CHG (costing £6.26).

\section{NICE Guidance}

\subsection{Provisional Recommendations}

The evidence submitted by the sponsor and the EAC's critique of this evidence was presented to the MTAC who provided draft recommendations relating to Tegaderm 
Table 4 Model input parameters used in EAC's update of the sponsor's economic model

\begin{tabular}{|c|c|c|c|}
\hline Variable & EAC point estimate & Source & EAC range and source \\
\hline Baseline CRBSI rate & $\begin{array}{l}\text { English data }(2010) \text { : } \\
1.48 \text { per } 1000 \text { catheter } \\
\text { days } \\
\text { Scottish data }(2013) \text { : } \\
0.3 \text { per } 1000 \text { catheter } \\
\text { days }\end{array}$ & $\begin{array}{l}\text { Bion et al. [6] } \\
\text { Scottish ICU report [36] }\end{array}$ & $\begin{array}{l}\text { DSA: range }=0.2-1.75 \text {, lower CI from Scotland } \\
\text { and upper CI from Bion et al. }[6,36] \\
\text { PSA: Gamma distribution. The PSA was run } \\
\text { twice, with English data and more recent } \\
\text { Scottish data } \\
\text { Range }=1.28-1.75,95 \% \text { CI from Bion et al. [6] } \\
\text { Range }=0.2-0.6,95 \% \text { CI from Scotland [36] }\end{array}$ \\
\hline $\begin{array}{l}\text { Hazard ratio for CRBSI } \\
\text { with Tegaderm } \mathrm{CHG}\end{array}$ & 0.402 & Timsit et al. [11] & $\begin{array}{l}\text { DSA: range is equal to } 95 \% \text { CI of } 0.186-0.868 \\
\text { PSA: lognormal distribution, with range equal to } \\
95 \% \text { CI of } 0.186-0.868 \text { [11] }\end{array}$ \\
\hline $\begin{array}{l}\text { Baseline local site infection } \\
\text { rate }\end{array}$ & $\begin{array}{l}0.14 \text { per } 1000 \text { catheter } \\
\text { days }\end{array}$ & NHS Wales 2013 data [33] & $\begin{array}{l}\text { DSA: a range of } 0-0.3 \text { infections per } 1000 \\
\text { catheter days } \\
\text { PSA: Gamma distribution, with an SE of } 0.1\end{array}$ \\
\hline $\begin{array}{l}\text { Hazard ratio for local site } \\
\text { infection with Tegaderm } \\
\text { CHG }\end{array}$ & 0.402 & $\begin{array}{l}\text { Assumed to be equal to hazard } \\
\text { ratio for CRBSI from Timsit } \\
\text { et al. [11] }\end{array}$ & $\begin{array}{l}\text { DSA: range equal to } 95 \% \mathrm{CI} \text { of } 0.186-0.868 \\
\text { PSA: lognormal distribution, with range equal to } \\
95 \% \text { CI of } 0.186-0.868 \text { [11] }\end{array}$ \\
\hline $\begin{array}{l}\text { Baseline dermatitis } \\
\text { probability }\end{array}$ & 0.0021 & Timsit et al. [11] & $\begin{array}{l}\text { DSA: a range of } 0-0.01 \\
\text { PSA: Beta distribution was used, with Alpha }=1 \\
\text { and Beta }=475[11]\end{array}$ \\
\hline $\begin{array}{l}\text { Risk reduction for } \\
\text { dermatitis with Tegaderm } \\
\text { CHG }\end{array}$ & 1 & Timsit et al. [11] & $\begin{array}{l}\text { DSA: a range of } \pm 100 \%(0-2) \\
\text { PSA: lognormal distribution, with an SE of } 0.5\end{array}$ \\
\hline Cost of CRBSI & $£ 9990$ & Hockenhull et al. [25] & $\begin{array}{l}\text { DSA: a range of } \pm 50 \%(£ 4950-14,850) \\
\text { PSA: Gamma distribution, with an SE of } £ 3000\end{array}$ \\
\hline Cost of dermatitis & $£ 6$ & Expert advice & $\begin{array}{l}\text { DSA: a range of } \pm 30 \%(£ 4.10-7.80) \\
\text { PSA: Gamma distribution, with an SE of } £ 3\end{array}$ \\
\hline Cost of local site infection & $£ 100$ & Expert advice & $\begin{array}{l}\text { DSA: a range of } \pm 30 \%(£ 70-130) \\
\text { PSA: Gamma distribution, with an SE of } £ 30\end{array}$ \\
\hline $\begin{array}{l}\text { Number of days with } \\
\text { catheter (catheter dwell } \\
\text { time) }\end{array}$ & 10 days & $\begin{array}{l}\text { Expert advice confirmation of Ho } \\
\text { and Litton [40] }\end{array}$ & $\begin{array}{l}\text { DSA: a range of } \pm 50 \% \text { ( } 5-15 \text { days) } \\
\text { PSA: Gamma distribution, with an SE of } 5 \text { days }\end{array}$ \\
\hline Number of dressings & 3 & $\begin{array}{l}\text { Assumption (based on change of } \\
\text { dressing every 3-7 days) }\end{array}$ & $\begin{array}{l}\text { DSA: a range of } \pm 66 \% \text { (1-5 dressings) } \\
\text { PSA: Gamma distribution, with an SE of } 2 \\
\text { dressings }\end{array}$ \\
\hline Cost of Tegaderm CHG & $£ 6.26$ & $\begin{array}{l}\text { NHS Supply Chain. Weighted } \\
\text { average of dressing sizes [35] }\end{array}$ & $\begin{array}{l}\text { This is fixed and not included in either the } \\
\text { deterministic or probabilistic analyses }\end{array}$ \\
\hline Cost of standard dressing & $£ 1.54$ & $\begin{array}{l}\text { NHS Supply Chain. Weighted } \\
\text { average of brands [35] }\end{array}$ & $\begin{array}{l}\text { This is fixed and not included in either the } \\
\text { deterministic or probabilistic analyses }\end{array}$ \\
\hline
\end{tabular}

$C H G$ chlorhexidine gluconate, $C R B S I$ catheter-related bloodstream infection, $C I$ confidence interval, DSA deterministic sensitivity analysis, EAC External Assessment Centre, ICU intensive care unit, NHS National Health Service, PSA probabilistic sensitivity analysis, SE standard error

CHG following their meeting in February 2015. These were as follows [37]:

1. The case for adopting the $3 \mathrm{M}$ Tegaderm $\mathrm{CHG}$ IV securement dressing for central venous and arterial catheter insertion sites is supported by the evidence. This technology allows observation and provides antiseptic coverage of the catheter insertion site, reducing catheter related bloodstream infections and local site infections compared with semipermeable transparent (standard) dressings. It can be used with existing care bundles.

2. The $3 \mathrm{M}$ Tegaderm CHG IV securement dressing should be considered for use in critically ill patients who need a central venous or arterial catheter in intensive care or high dependency units. 
3. The estimated cost saving from using a $3 \mathrm{M}$ Tegaderm CHG IV securement dressing (Tegaderm CHG) instead of a standard transparent semipermeable dressing is $£ 73$ per patient. These estimates are based on a baseline catheter-related bloodstream infection rate of 1.48 per 1000 catheter days. Tegaderm CHG is estimated to be cost neutral when the baseline catheterrelated bloodstream infection rate is 0.24 per 1000 catheter days, and cost incurring when the baseline rate falls below that figure. Estimates of the benefiting population vary from around 88,000 to 226,000 depending on whether adult ICU episodes longer than 48 hours requiring a central venous catheter, or all adult ICU episodes requiring a central venous catheter, are used. Based on these estimates, if the use of Tegaderm CHG became standard practice, it has the potential to save the English NHS between $£ 6.2$ million and $£ 16.5$ million each year, assuming the baseline catheter related bloodstream infection rate is 1.48 per 1000 catheter days [37].

\subsection{Consultation Response}

During the consultation, NICE received 44 consultation comments from seven consultees. Consequently, the NICE draft guidance was updated to address these comments. A description of central-line-associated bloodstream infection (CLABSI) was added, along with an explanation of its association to CRBSI.

The epic3 guideline was used within the Tegaderm CHG draft guidance to describe current best practice guidance for preventing healthcare-associated infections in English NHS hospitals [38]. The description of the evidence that the epic3 guideline is based upon was expanded in the final NICE guidance to include more detail on the quality of this evidence. In regard to the evidence included in the sponsor's submission, the guidance was updated to describe where this submission deviated from the original scope; the key deviation being the lack of comparison between Tegaderm CHG and other CHG-impregnated dressings.

Other sections of the guideline were updated to describe in more detail the benefits of visualising the catheter insertion site and to clarify that the recommendations relate to adults only. Minor revisions were made to other sections to improve clarity.

There were no consultation comments about the estimated potential population-level cost savings in draft recommendation 3; however, further work by the NICE Resource Impact Assessment team resulted in revisions to this section. In particular, there was a change to the estimated uptake of the technology. The section is based on an estimate of current use of $15 \%$, and assumes future uptake at $80 \%$.

\section{Key Challenges and Learning points}

The key challenges faced by the EAC and the sponsor were the lack of evidence comparing Tegaderm CHG with other CHG-impregnated dressings and the generalisability of the clinical evidence. As none of the clinical studies included by the sponsor or identified by the EAC compared Tegaderm CHG and other CHG-impregnated dressings, the EAC had to rely on an informal indirect comparison of the two dressings using the two RCTs published by Timsit et al. [11, 12]. The similar inclusion criteria, outcome measurement and patient characteristics between the two studies aided confidence in the comparison [11, 12]. A cost-minimisation exercise was conducted by the EAC to compare Tegaderm CHG and other CHG-impregnated dressings based upon the assumption of clinical equivalence. This exercise provided an estimate of the relative cost effectiveness of the two dressings. However, the lack of comparative data prevented both the sponsor and EAC conducting a robust cost-effectiveness comparison.

Three of the four clinical studies included by the EAC were set outside the UK $[11,12,20]$. Catheter insertion site care protocols and patients included within the studies differed in part to those in the UK. The EAC assessed the generalisability of the studies to the NHS by consulting with clinical experts and making comparisons with English clinical guidelines. Two of the three studies were judged to have sufficient external validity to address the scope of the decision problem [11, 12], whilst the third did not include enough information to make a reliable judgement [20].

The key challenge of the economic evaluation related to the variation in measurement and recording of baseline CRBSI between hospitals. The sponsor utilised a rate of 1.48 CRBSI per 1000 catheter days within their economic modelling. As described in Sect. 4.2.3, the EAC undertook scenario and sensitivity analysis around this value. The difficulties associated with measuring CRBSI and the implications of this were captured in the final guideline.

The assessment process was aided by the good quality of the sponsor's submission, which included robust literature searches for clinical evidence and model input parameter values as well as robust economic modelling with probabilistic analysis. Where possible, if not provided by the sponsor, the EAC should undertake probabilistic sensitivity analyses in order to capture uncertainty within the economic modelling results. However, the benefits of undertaking the analyses are dependent upon the information available to inform confidence around input parameters. 
Where there is a paucity of evidence, the reliability of the probabilistic analysis results will be compromised.

\section{Conclusion}

The MTEP evaluation process was followed for the development of medical technologies guidance on Tegaderm CHG. This included a submission of clinical and economic evidence by the sponsor, critical appraisal of this evidence by the EAC, additional work to address remaining uncertainties, drafting of recommendations by the MTAC, and a subsequent consultation. Following this process, the MTAC judged that the evidence demonstrated sufficient potential benefits of Tegaderm CHG to patients and the NHS to allow positive recommendations to be made for the dressing.

Acknowledgments The authors are grateful to the clinical experts identified by NICE, who provided expertise and clinical knowledge. Newcastle upon Tyne Hospitals and York Health Economics Consortium are funded by NICE to act as an EAC for the MTEP. This summary of the Medical Technology Guidance was produced following publication of the final guidance report. This summary has not been externally peer reviewed by Applied Health Economics and Health Policy. One of the authors (A.S.) is an NHS employee; the NHS has a financial interest in the guidance issued by NICE as a result of this work. Four of the authors (M.J., W.G., M.A., J.C.) work for the EAC, but otherwise have no conflicts of interest. N.H. is an employee of NICE.

Author contributions The manuscript was prepared by M.J., with contributions from J.C., W.G., N.H., M.A. and A.S. Literature searching was undertaken by M.A. and evidence review by M.J. and W.G., with advice and quality assurance from J.C. The model critique was undertaken by M.J., with advice and quality assurance from J.C. N.H. reported the decision problem and the NICE recommendations. The guarantor for overall content is A.S.

Open Access This article is distributed under the terms of the Creative Commons Attribution-NonCommercial 4.0 International License (http://creativecommons.org/licenses/by-nc/4.0/), which permits any noncommercial use, distribution, and reproduction in any medium, provided you give appropriate credit to the original author(s) and the source, provide a link to the Creative Commons license, and indicate if changes were made.

\section{References}

1. National Institute for Health and Care Excellence. The 3M Tegaderm CHG IV securement dressing for central venous and arterial catheter insertion sites. 2015. https://www.nice.org.uk/ guidance/MTG25. Accessed 23 July 2015.

2. Campbell B, Campbell M. NICE medical technologies guidance: a novel and rigorous methodology to address a new health technology assessment challenge. Appl Health Econ Health Policy. 2012;10(5):295-7.
3. $3 \mathrm{M}$. Instructions For Use: $3 \mathrm{M}^{\mathrm{TM}}$ Tegaderm ${ }^{\mathrm{TM}} \mathrm{CHG}$ Chlorhexidine Gluconate I.V. Securement Dressing [obtained via sponsor's submission]. 2012.

4. Health and Social Care Information Centre. Hospital Episode Statistics: Adult Critical Care in England April 2012 to March 2013. 2014. http://www.hscic.gov.uk/catalogue/PUB13893/adulcrit-care-data-eng-apr-12-mar-13-rep.pdf. Accessed 23 Dec 2014.

5. Curtis RL. Catheter-related bloodstream infection in the intensive care unit. J Intensive Care Soc. 2009;10(2):102-8.

6. Bion JF, Richardson A, Hibbert P, et al. 'Matching Michigan': a 2-year stepped interventional programme to minimise central venous catheterblood stream infections in intensive care units in England. BMJ Qual Saf. 2013;22:110-23.

7. Shah H, Bosch W, Thompson KM, Hellinger WC. Intravascular catheter-related bloodstream infection. Neurohospitalist. 2013;3(3):144-51.

8. Fletcher S. Catheter-related bloodstream infection. Contin Educ Anaesth Crit Care Pain. 2005;5(2):49-51.

9. National Institute for Health and Care Excellence. Infection: prevention and control of healthcare-associated infections in primary and community care. 2012. https://www.nice.org.uk/ guidance/cg139. Accessed 12 Dec 2014.

10. National Institute for Health and Care Excellence. The $3 \mathrm{M}$ Tegaderm CHG IV securement dressing for cnetral venous and arterial catheter insertion sites: final scope. 2014. https://www. nice.org.uk/guidance/gid-mt238/documents/the-3m-tegaderm-chgiv-securement-dressing-for-central-venous-and-arterial-catheterinsertion-sites-final-scope2. Accessed 9 Apr 2015.

11. Timsit JF, Mimoz O, Mourvillier B, Souweine B, GarrousteOrgeas M, Alfandari S, et al. Randomized controlled trial of chlorhexidine dressing and highly adhesive dressing for preventing catheter-related infections in critically ill adults. Am J Respir Crit Care Med. 2012;186(12):1272-8.

12. Timsit JF, Schwebel C, Bouadma L, Geffroy A, GarrousteOrgeas M, Pease S, et al. Chlorhexidine-impregnated sponges and less frequent dressing changes for prevention of catheter-related infections in critically ill adults: a randomized controlled trial. JAMA. 2009;301(12):1231-41.

13. National Institute for Health and Care Excellence. Medical Technologies Evaluation Programme: Process Guide. 2011. http://www.nice.org.uk/Media/Default/About/what-we-do/NICEguidance/NICE-medical-technologies/Medical-technologiesevaluation-programme-process-guide.pdf. Accessed 6 Feb 2015.

14. Bashir MH, Olson LK, Walters SA. Suppression of regrowth of normal skin flora under chlorhexidine gluconate dressings applied to chlorhexidine gluconate-prepped skin. Am J Infect Control. 2012;40(4):344-8.

15. Maryniak K. Clinical performance and nursing satisfaction of a transparent chlorhexidine gluconate IV securement dressing with peripherally inserted central catheters. J Assoc Vasc Access. 2009;14(4):200-3.

16. Olson C, Heilman JM. Clinical performance of a new transparent chlorhexidine gluconate central venous catheter dressing. J Assoc Vasc Access. 2008;13(1):13-9.

17. Rupp ME, Cavalieri J, Delaney K, Lundgren K, Stammers L, Beach S, editors. Prospective, randomized, controlled trial assessing the clinical performance of a transparent chlorhexidine gel pad intravascular catheter dressing. 18th Annual Meeting of the Society for Healthcare Epidemiology of America; 5th-8th April 2008; Orlando.

18. Moher D, Liberati A, Tetzlaff J, Altman DG. Preferred reporting items for systematic reviews and meta-analyses: the PRISMA statement. Br Med J. 2009;339(7716):332 (D. A.).

19. Karpanen T, Casey A, Nightingale P, Whitehouse T, Das I, Elliott TSJ, editors. A clinical study on the antimicrobial efficacy of a 
transparent chlorhexidine gel pad intravascular catheter dressing. Hospital Infection Society Conference; 16-18th Nov 2014; Lyon.

20. Roberts B, Cheung D. Biopatch-a new concept in antimicrobial dressings for invasive devices. Aust Crit Care. 1998;11(1):16-9.

21. Deschneau M, Pyrek J, Moureau NL, editors. A Multicenter prospective open label evaluation of the clinical performance of a chlorhexidine gluconate antimicrobial transparent dressing. Infusion Nurses Society Conference; 2008.

22. Eyberg CI, Pyrek J. A controlled randomized prospective comparative pilot study to evaluate the ease of use of a transparent chlorhexidine gluconate gel dressing versus a chlorhexidine gluconate disk in healthy volunteers. J Assoc Vasc Access. 2008;13(3):112-7.

23. Zehrer CL, Smith GD, Deschneau M, editors. Evaluation of a new CHG gel pad dressing for catheter care. Infusion Nursing Society; 16th-21st May 2009; Nashville.

24. Crawford AGF, Joseph P Jr, Rao B Jr. Cost-benefit analysis of chlorhexidine gluconate dressing in the prevention of catheterrelated bloodstream infections. Infect Control Hosp Epidemiol. 2004;25(8):668-74.

25. Hockenhull JC, Dwan K, Boland A, Smith G, Bagust A. The clinical effectiveness and cost-effectiveness of central venous catheters treated with anti-infective agents in preventing bloodstream infections: a systematic review and economic evaluation. Health Technol Assess. 2008;12(12):154.

26. Schwebel C, Lucet JC, Vesin A, Arrault X, Calvino-Gunther S, Bouadma L, Timsit, JF. Economic evaluation of chlorhexidineimpregnated sponges for preventing catheter-related infections in critically ill adults in the Dressing Study. Crit Care Med. 2012;40(1):11-7.

27. Veenstra DL, Sain S, Sullivan SD. Cost-effectiveness of antiseptic-impregnated central venous catheters for the prevention of catheter-related bloodstream infection. J Am Med Assoc. 1999;282:554-60.

28. Ye X, Rupnow M, Bastide P, Lafuma A, Ovington L, Jarvis WR. Economic impact of use of chlorhexidine-impregnated sponge dressing for prevention of central line-associated infections in the United States. Am J Infect Control. 2011;39(8):647-54.

29. Maunoury F, Motrunich A, Palka-Santini M. Cost-effectiveness modeling of antimicrobial dressings for preventing catheter-related bloodstream infection: Homogeneous versus non-homogeneous Markov approaches. Value Health. 2014;17(7):A564.

30. Maunoury F, Motrunich A, Ruckly S, Timsit JF. Non-homogeneous cost-effectiveness modeling of a new chgdressing for preventing catheter-related bloodstream infections for patients in intensive care units. Value Health. 2013;16(7):A356.
31. Palka-Santini M, Maunoury F, Motrunich A, Ruckly S, Timsit JF. Cost-effectiveness analysis of a new CHG-dressing for preventing catheter-related bloodstream infections. J Vasc Access. 2014;15(3):229-30.

32. Palka-Santini M, Motrunich A, Maunoury F. Cost-effectiveness analysis of an antimicrobial transparent dressing for protecting central vascular accesses in critically ill patients versus standard transparent dressings in France: a comparison of two modeling approaches: decision-tree versus non-homogeneous Markov model. Value in Health. 2014;17(7):A560.

33. Welsh Healthcare Associated Infection Programme. Central Venous Catheter (CVC) Infection and Ventilator Associated Pneumonia (VAP) in Critical Care-Annual Report. 2014. http:// www2.nphs.wales.nhs.uk:8080/WHAIPDocs.nsf/(\$All)/28459C 38515ACCAC80257D79005253C4/\$File/Aneurin\%20Bevan\%20 Critical\%20Care\%20CVC\%20\%26\%20VAP\%20Report\% 202013. pdf?OpenElement. Accessed 8 Jan 2015.

34. Pemberton LB, Ross V, Cuddy P, Kremer H, Fessler T, McGurk E. No difference in catheter sepsis between standard and antiseptic central venous catheters. A prospective randomized trial. Arch Surg. 1996;131(9):986-9.

35. NHS. NHS Supply Chain Website. http://www.supplychain.nhs. uk/. Accessed 7 Jan 2015.

36. Health Protection Scotland. Surveillance of Healthcare Associated Infections in Scottish Intensive Care Units. Annual report of data from January-December 2013. 2014. http://www. documents.hps.scot.nhs.uk/hai/sshaip/publications/icu-surveillan ce/icu-annual-report-2014.pdf. Accessed 6 Jan 2015.

37. National Institute for Health and Care Excellence. Medical Technologies Consultation Document: The 3M Tegaderm CHG IV securement dressing for central venous and arterial catheter insertion sites. 2015. https://www.nice.org.uk/guidance/gid-mt 238/documents/the-3m-tegaderm-chg-iv-securement-dressing-forcentral-venous-and-arterial-catheter-insertion-sites-consultationdocument2. Accessed 14 May 2015.

38. Loveday HP, Wilson JA, Pratt RJ, Golsorkhi M, Tingle A, Bak A, et al. epic3: national evidence-based guidelines for preventing healthcare-associated infections in NHS hospitals in England. J Hosp Infect. 2014;86(Suppl 1):S1-70.

39. Saint S, Veenstra DL, Lipsky BA. The clinical and economic consequences of nosocomial central venous catheter-related infection-are antimicrobial catheters useful? Infect Control Hosp Epidemiol. 2000;21(6):375-80.

40. Ho KM, Litton E. Use of chlorhexidine-impregnated dressing to prevent vascular and epidural catheter colonization and infection: a meta-analysis. J Antimicrob Chemother. 2006;58(2):281-7. 